\title{
Cambios histológicos del ligamento periodontal y hueso alveolar frente a fuerzas ortodónticas ligeras (estudio piloto)
}

\section{ARTículo Original}

Histological changes of the periodontal ligament and alveolar bone in front to light orthodontic forces (pilot study)

\section{Luciano Soldevilla Galarza ${ }^{1 *}$; Luis H.} Gálvez Calla²

1 Departamento Académico de Estomatología Pediátrica

2 Instituto de Investigación Estomatológica, Deparamento Académico de Ciencias Básicas * Facultad de Odontología Universidad Nacional Mavor de Sar Marcos, Lima, Perú E-mail: lucianosol@yahoo.com

\begin{abstract}
Resumen
El hueso maxilar al igual que el ligamento periodontal experimentan cambios constantes cuando se emplean fuerzas leves, sin embargo, son persistentes durante los tratamicntos ortodónticos. El diente es tratado como cucrpo deformable que se separa del ligamento periodontal y del hueso $0^{1,3,4}$. La formación y resorción ósea ocurren de manera continua, pero existe un equilibrio fisiológico entre ambos. En condiciones normales, el hueso sufre constante resorción y en otras se conserva el estado rcgular de ese momento. El estado fisiológico del hueso depende de la edad y sus funciones. Está claro que la velocidad de resorción y aposición de hueso dependerá de la respuesta establecida por la función; sin embargo, durante el movimicnto dentario a través del hueso por fuerzas ortodónticas, el espesor del ligamento permanece en un valor constante
\end{abstract}

\section{A bstract}

The maxillary bone the same as the periodontal ligament experiences constant changes when light forces are used, however, they are persistent during the orthodontic treatments. The tooth is treated as body deformable that separates the periodontal ligament and of the bone ${ }^{1,3,1}$. The formation and bony resorption happen in a continuous way, but a physiclogic balance exists between both Under normal conditions, the bone suffers constant resorption and in other the regular state of that moment is conserved. 'The physiologic state of the bone depends on the age and its functions. It is clear that the speed of resorption and bone apposition will depend on the answer settled down by the function; however, during the movement dentario through the bone for orthodontic forces, the thickness of the ligament remains in a constant value.
Palabras clave: fuerzas ortodónticas ligeras, tensión, presión, histología dentoalveolar

Key words: light orthodontic forces, tension, pressure, dentoalvenlar histology

\section{Introducción}

\section{Reacciones tisulares frente a las} fuerzas ortodónticas

Los dientes se encuentran unidos al proceso alveolar a través de la arliculación alveolo dentaria, que constituye el periodonto de inserción; representado por el cemento, ligamento periodontal y hueso alveolar ${ }^{\downarrow}$

Las fucrzas leves y persistentes aplicadas durante la terapéutica ortodonlica generan cambios biológicos en tos tejidos dentoalveolares y consecuentemente el movimiento ortodóntico correspondicnte. Los tcjidos involucrados, tales como:

El ligamento periodontal; de $0,5 \mathrm{~mm}$. que ocupa el espacio comprendido entre la pared del alveolo y el cemento dentario, esta constituido por fibras colágenas insertadas de un lado en el cemento radicular y del otro en el hueso alveolar, entremezcladas con vasos sanguíneos, clementos celulares, terminaciones nerviosas y liquicio intersticial; que en su conjunto forman un eficaz sistema amortiguador y disipador de fuerzas fisiologicas aplicadas en un breve intervalo de tiempo durante las funciones oclusales, siendo su función principal la de fijar, a través de sus fibras colágenas, al hueso alveolar.

La proliferación de elementos celulares está presente durante los estímulos funcionales para modificar la posición espacial del diente ${ }^{1}$ El tipo de colágeno expresado es de tipo XII, relacionado con el desarrollo, orga- nización y la alineación de las fibras periodontales del ligamento. Además, el colágeno de tipo VI ha sido immunolocalizado en el ligamento periodontal y gíngiva, y parece estar ligado a estructuras microfibrilares pequeñas de colágeno y elastina². La matriz extracelular propurciona funciones importantes a los tejidos blandos conectivos del periodonto manteniendo la integridad y la regulación estrurtural de las funciones celulares ${ }^{2}$

Los vasos sanguíneos son los responsables de la nutrición del ligamento periodontal, sirven de vía de acceso de las células responsables de la remodelación del hueso cortical y ligamentos ${ }^{1}$.

La resorción y aposición del hueso alveolar puede suceder de modo si- 
multáneo. La fosfatasa alcalina es un biomarcador indicativo de formación ósea, se observa aumentado durante las primeras 3 semanas del tratamiento ortodóntico; mientras que la fosfatasa ácida se encuentra entre la 3ra y la 6ta semana subsecuentes al tratamiento, por lo que se le reconoce como marcador importante de la actividad del osteoclasto y de la resorción del hueso ${ }^{2}$.

Es probable que el cemento Radicular al no estar vascularizado sea escasamente modificado por los estímulos masticatorios o por cargas de presión $y$ tensión, y por lo tanto no este involucrado en los mecarismos reactivos provenientes del tratamiento ortodóntico; siendo el ligamento periodontal y hueso alveolar los principales elementos de evaluación ${ }^{\mathrm{j}-3}$

El periodonto de inserción cumple un rol importante en la estabilización del diente durante los esfuerzos funcionales; cuando estas fuerzas son aplicadas sobre el elemento dentario, este se disloca en el interior del espacio alveolar, produciéndose el estiramiento de algunas fibras periodon. tales y la comprestón de otras, simultáneamente el liquido que llena los espacios entre las fibras tambien es comprimido contra las paredes óseas. La falta de drenaje de los fluidos dificulta el movimiento dentario, ejerciendo una resistencia hidráulica al movimiento. Las fibras periodontales y el liquido intersticial actuan en conjunto, contraponiéndose a las cargas aplicadas sobre el diente, haciendo que vuelva a su posición original. Este proceso ocurrirâ siempre y cuando el periodo de fuerza sea de corta duración.

\section{Respuesta ortodóntica ideal}

El hueso alveolar debido a su plasticidad se adapta a las fuerzas funciona les que actúan sobre el, depositando hueso en áreas sometidas a fuerzas de tracción y reabsorbiendo tejido óseo las áreas donde hay presión. El movimiento ortodóntico solo es posible por este mecanismo ${ }^{4}$

En las primeras fracciones de segundo, la fuerza ortodóntica intenta dislocar la raíz dentaria contra el alvéom lo, siendo impididas por las fibras periodontales y el efecto hidráulico del liquido intersticial, transfiriéndose la carga al hueso alveolar, crean do el llamado efecto piezoeléctrico; que consiste en un flujo de electro- nes que se dislocan de la malla cristalina de un hueso en remodelación. Algunos autores suponen que este efecto tiene influencia en los proceso de movimiento dentario porque los campos eléctricos alteran la permeabilidad de la membrana celular ${ }^{3,4}$

Después de las 24 horas del uso de la fuerza ortodontica, los primeros segundos hasta el segundo día, fase inicial del movimiento ortodon-tico, comienzan tas reacciones celulares ${ }^{3}$ el liquido intersticial drena a los tejidos vecinos, debido a la porosidad del hueso alveolar, dejando de ejercer la mencionada presión hidráulica que evita la dislocación radicular. De esta forma la raiz se aproxima mas todavía a la pared del alvéolo dis. tendiendo los ligamentos periodontales del lado en que se aplicó la fuerza y comprimiendo aquellos del lado opuesto ${ }^{14}$. El sistema vascular que ocupa el $50 \%$ del espacio periodontal es comprimido lo que dificulta la circulación sanguínea provocando un proceso isquémico, fanto de lado de tensión como del lado de compresión ${ }^{5,4}$. La respuesta del tejido es similar a un proceso inflamatorio, la histamina liberada por los mastocizos de la región agredida tiene acción inmediata sobre los va. sos sanguineos produciendo vasodilatación, abriendo espacios entre las células endoteliales que forman sus paredes lo que provoca un aumento de la permeabilidad. Esta primera reacción local recibe el nombre de respuesta inmediata.

Algunas protefnas que se encuentra normalmente presentes en la circulación sanguínea son liberadas al interior de los tejidos periodontales para producir cininas (principalmente bradicidina) en reemplazo de la histamina para mantener el proceso inflamatorio ${ }^{2}$ La agresión a las membranas celulares induce la formacion de prostaglandinas, cuya acción junto con las cininas será preservar la vasodilatación y el aumento de la permeabilidad vascular. El incremento de la irrigación sanguinea posibilita el aumento de la actividad metabólica celular lo que será de gran importancia para los procesos remodeladores siguientes, denominada respuesta tardia, que ocurre lue go de 2 a 4 horas después de la aplicación de la fuerza ortodóntica, permaneciendo activa mientras dure el estímulo. La vasodilatación propicia la salida de monocitos, de cuya fusión resultan las células multinuclea* das conocidas como osteoclastos, responsables de la reabsorción de la cortical alveolar adyacente a la compresión de ligamentos. En el lado opuesto donde hay distensión de los ligamentos se estimulan las células mesenquimales indiferenciadas, transformándose en osteoblastos y fibroblastos.

Estudios experimentales in vitro han demostrado que los fibroblastos aplanados sintetizan más $A D N$ que la cé lula redondeadas que son más catabólicas. De manera que las células aplanadas propias de áreas de tensión inducen mecanismos de sintesis; mientras que las células redondeadas en áreas de presión propician degradación de la matriz exiracelular ${ }^{2}$. Clínicamente este periodo se caracteriza por dolor moderado en los dientes sometidos a carga, pero sin que estos se movilicen.

Otros estudios in vivo que evalúan la comprestón y tensión en el ligamento periodontal, han indicado que la actividad metabólica es similar en los lados de presión y de tensión de los dientes sometidos a una fuerza ortodóntica ${ }^{2,4}$

Después del segundo día de la aplicación de fuerzas, las modificaciones locales permiten que los osteoclastos y osteoblastos inicien los procesos de remodelación ósea, con aposición en el lado de tensión de las fibras periodontales y reabsorción en el lado dè compresión de los mismos. Lentamente el alvéolo se disloca en el sentido de la aplicación de la fuerza con el consecuente movimiento ortodóntico ${ }^{4}$.

\section{Factores que intervienen en la respuesta ortodóntica}

Por ser el movimiento ortodónfico un proceso complejo que involucra a di ferentes tejidos, como: hueso alveolar, fibras colágenas del ligamento periodontal y vasos sanguíneos, la respuesta del diente a la fuerza ortodóntica estará supeditada a diversos factores:

\section{Magnitud de fuerzas:}

Fuerzas inocuas, son aquellas fuerzas de pequeña magnitud que son incapaces de iniciar el efecto electroquímico responsable del movimiento ortodóntico.

Fuerzas leves, son pequeñas fuerzas con que se inicia el movimiento dentario hasta alcanzar una fuerza óptima, 
con el cual se produce el movimiento ortodóntico mas eficaz ${ }^{1,4}$, sin molestias para el paciente y sin dano tisular; pérdida o resorción radicular. Generalmente, se cree que el uso de un sistema óptimo de fuerzas es importante para una respuesta biológica adecuada en el sistema periodontal $1^{3,4}$

Cuando aplicamos una fuerza óptima sobre los ligamentos periodontales, la compresión y la distensión de sus fibras preservará su vitalidad en toda su extensión e iniciará una respuesta celular de aposición y resorción ósea de intensidad máxima. Esta fuerza optima provoca rá resorción de las paredes alveolares o resorción frontal y será la fuerza ortodóntica mas fisiológica.

Si aumentamos la carga sobre el diente observaremos que en algunas ares del ligamento periodontal habrá mayor concentración de tensiones con una presión excesiva en sus tejidos. En estas regiones, en general, en el lado de compresión del ligamento, la circulación sanguínea se volverá lenta o casi nula, ocasionando la degeneración o necrosis aseptica de las fibras periodontales (fenómeno de hialinizacion $)^{4.5}$. Las árcas hialinizadas retrasan el movimiento dentario e incluso dificultan la inmovilización del diente, ya que un tejido conjuntivo saludable es indispensable para la remodelación ósea. Cuanto más áreas hialinizadas existan más lento será el movimiento ortodóntico; por lo tanto, cuanto más intensa es la fuerza menos será la velocidad de migración dentaria ${ }^{1}$.

Las áreas de hialinización son fagocitadas por las células gigantes a cuerpo extraño, macrófagos y neutrófilos, solo así es posible reanudar el movimiento del diente. Luego de la limpieza histica, fase indispensable de todo proceso reparativo, se produce una resorción laminar por los osteoclastos comenzando la lase de la aceleración y dislocación ortodóntica apropiada. En ausencia de áreas necróticas, el fibroblasto y el cementoblasto son las encargadas de iniciar el proceso de remodelación del hueso en el lado de tensión, elevando el índice del movimiento dentario ${ }^{3-6}$

En estudios experimentales ${ }^{3,5}$ se han observado una acumulos de osteoclastos presentes en la vecindad de las área de hialinización, en algunos casos los osteoclastos estaban implicados en resorción directa del hueso

Cuadro 1

\begin{tabular}{|c|c|c|}
\hline & FUERZAS LEVES & FUERZAS PESADAS \\
\hline Tipo je resorcion & $\begin{array}{l}\text { Fronitil } \\
\text { fon in pored dxe nuecol }\end{array}$ & $\begin{array}{l}\text { Minnate oa } \\
\text { cistorsiz }\end{array}$ \\
\hline Alencrionnes tisulores & 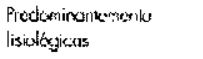 & 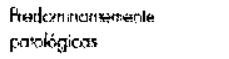 \\
\hline $\begin{array}{l}\text { lipo de morvizienta } \\
\text { dentotio }\end{array}$ & Conitines & Inkermilente \\
\hline 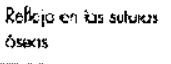 & Pequerios & 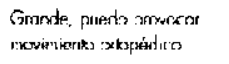 \\
\hline $\begin{array}{l}\text { Sensurtity: } \\
\text { ctaleressi }\end{array}$ & $\begin{array}{l}\text { presante an kis srimeras } \\
203 \text { dias }\end{array}$ & 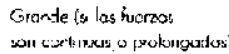 \\
\hline
\end{tabular}

pero más a menudo en resorciones radiculares. En los lados de tensión ocurre aposición ósea, cubierta principalmente de las células osteoblásticas. Algunos casos registran una delgada capa de material osteoide depositada en las fibras de Sharpey ${ }^{3,5}$. El colágeno presente en tejidos conectivos mineralizados es de tipo I, que cumple un rol importante en el deposito cristalino del fosfato de cal$\mathrm{cio}^{2}$

Pilon et $a 1^{5}$ en sus estudios experimentales estandardizados en perros, utiljzaron fuerzas de 50,100, o $200 \mathrm{~N}$ para mover a los segundos premolares inferiores, que generan presiones localizadas de $10,20,040 \mathrm{kPa}$, que representan presiones bajas, moderadas, o altas, respectivamente. El desarrollo de áreas hialinizadas podia desempenar un papel importante en estas variaciones ${ }^{5.5}$. Estudios recientes sugieren que el desarrollo y la limpieza del tejido blando necrótico es un proceso continuo durante la dislocación del diente y no un solo acontecimiento ${ }^{5}$.

Las Fuerzas pesadas, son aquellas que producen grandes áreas de hialinización en la zona de compresión del ligamento periodontal, que dificulta la resorción frontal de la lamina dura del alveolo, manteniéndose inmóvil el diente por un largo período de tiempo. Los cortes histoló-gicos muestran necrosis aséptica del tejido periodontal en la zona de compresión de los ligamentos asociado a oclusión vascular, falta de suministro sanguíneo y anoxia de las células conjuntivas. La presión excesiva en la superficie cortical del alveolo disipada hacia el interior del hueso basal, cuyo gradiente de fuerza es capaz de producir estímulos químicos y eléctricus responsables de la resorción ósea respectiva, denominada resorción ósea a distancia o resorcion minante.
Cuando las fuerzas pesadas se mantienen por unos dias, la reabsorción ósea crece progresivamente hasta comprometer la cortical alveolar $y$ dislocar el diente, y en consecuencia se produce otro movimiento ${ }^{1,4}$.

Las células son los protagonistas principales de la remodelación de los tejidos blandos y duros y la reacción a los cambios tensionales de las fuerzas aplicadas ${ }^{5.4}$. Cuadro 1.

\section{Material y métodos}

Se utilizó un grupo de 4 conejos de la misma camada, cuyas edades eran de aproximadamente 12 meses. Los animales nacieron y crecieron hasta el momento de iniciarse el proceso de investigación, en el bioterio de la Facultad de Medicina Veterinaria de la UNMSM. Se alimentaron de un concentrado comercial (conejina) y de al. falfa.

Ins conejos fueron sometidos a una fuerza ortodúntica con anclajes reciprocos en los dos incisivos centrales inferiores, aplicados con el propósito de lograr la separación de estos dientes. Para tal efecto, se colocaron dos bandas de acero inoxidable con brackets soldados (ranura 0.022") en los incisivos centrales inferiores, con un alambre $0.017^{\prime \prime} \times 0.025^{\prime \prime}$ SS y un resorte $\mathrm{NiTi}$ comprimido que ejercíó una fuerza de 1.5 onzas (medida con onzímetro), sujetados con módulos elastoméricos.

Los especimenes fueron sacrificados después de $0,7,14$ y 21 días respectivamente. Se prepararon las muestras para su observación en cortes histológicos teñidos con hematoxilina eosina. 


\section{Resultados}

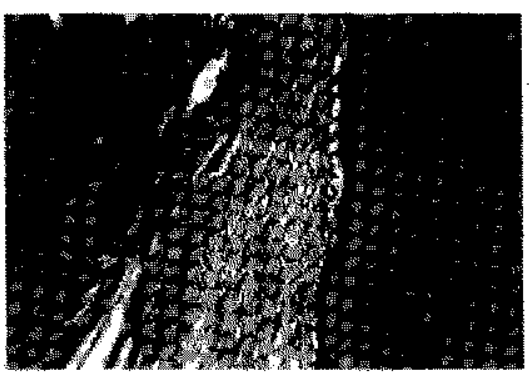

Fig. 1 A los 7 días se observa proliferación de células en la zona de tensión

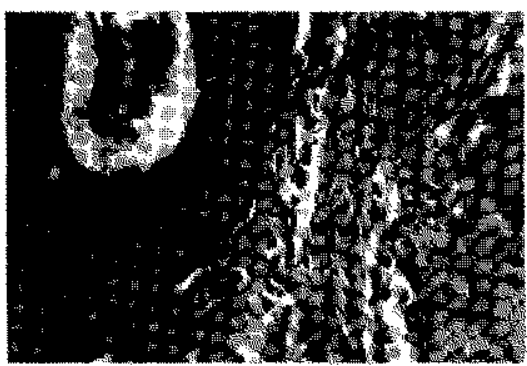

Fig. 3: A los 7 dias se observa celulas mesenquimales que se transtormaxán en fibroblastos y osteoblastos

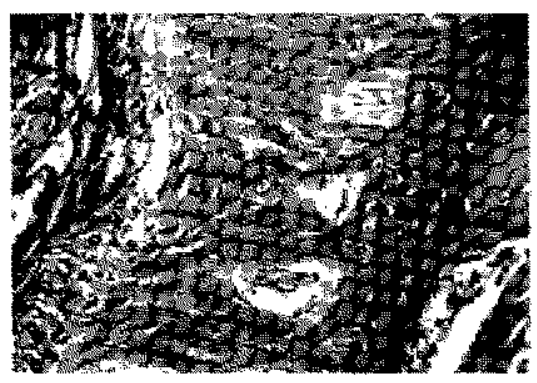

Fig.5: Proliferacion en la zona de tensión, formación osea

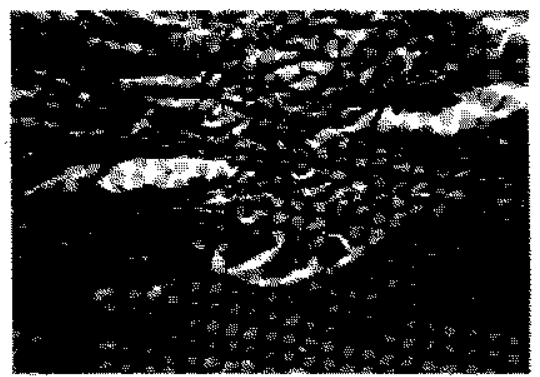

Fig.7: A los 14 dias se observa en la zona de compresión osteroclastos y vasodila-tación

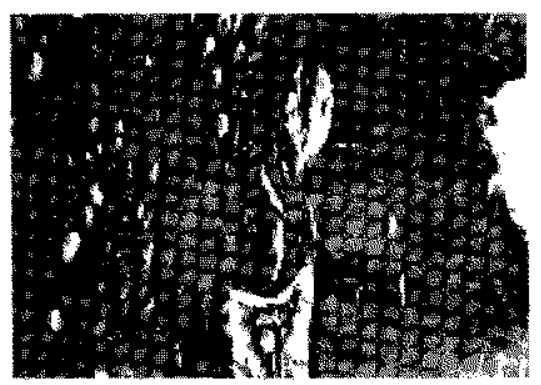

Fig. 2: A los 7 dias observamos perdida de celulas en la zona de compresión y hacia la izquierda la zona de tension con cellulas.

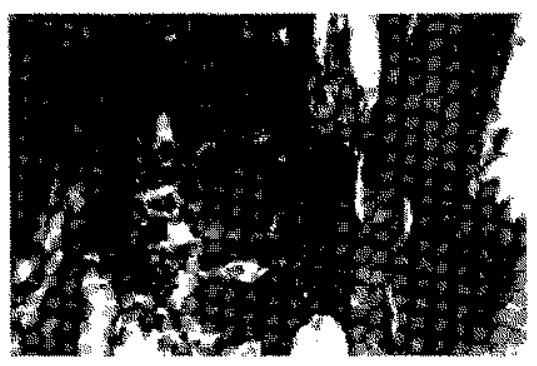

Fig. 4: Se observan fibras de sharpey, que son haces de colageno en la zona de terusión.

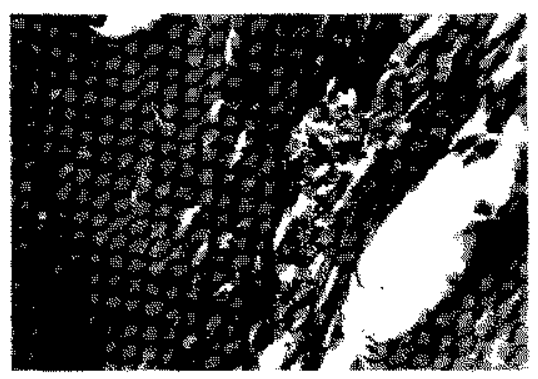

Fig.b: A los 14 dias se observan en la parte central escotaduras de reabsorcion conocidas como Lagunas de Howship que son zonas de reabsorción ósea, en esta fotografia no se ven los osteoclastos por que deben encontrarse en un plano mucho mas profundo.

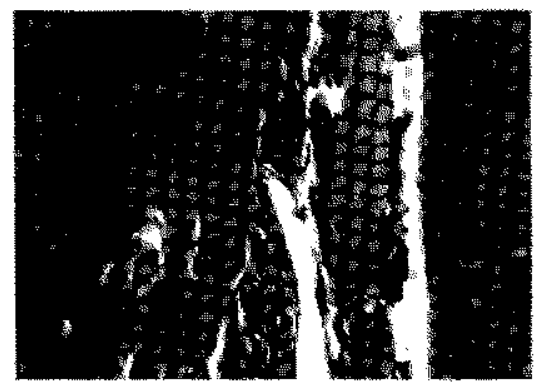

Fig. 8: Se observa en el centro del LPD la zona de Hialínización presentăndose hueso amorfo sin célulalas 


\section{Discusión}

A fines del siglo XIX, cada vez fue mayor el número de investigaciones en animales ${ }^{78}$, sobretodo referido a movimientos ortodónticos en ratas, utilizando fuerzas relativamente altas ${ }^{9,10}$; no obstante que las ratas como modelo experimental tiene serias desventajas.

Los roedores tiemen crupción continua en sus incisivos y migración mesial fisiológica en sus molares. La erupción continua de los incisivos puede afectar la dirección de la fuerza aplicada debido a que estos se utilizan a menudo como la tuidad de anclaje en estos experimentos. Utilizando el perro beagle como modelo se podría superar estos problemas 5 .

Los resultados obtenidos en este estudio piloto son comparables con los resultados obtenidos en otras investigaciones, sobre todo, relativos a los cambios histológicos del hueso alveolar y del ligamento periodontal: aunque diferentes en grado, debido al uso de diversos aparatos ortodónticos y a las diversas magnitudes, tipos y duración de fuerzas ${ }^{11}$

\section{Conclusiones}

- En términos generales, en el lado de compresión del ligamento periodontal, se observan áreas degenerativas o de necrosis aséptica que comprometen elementos fibrilares, con nula o escasa vascularización. (áreas de hialinización). Aseverando que, cuanto mayor es el ârea de hialinización mas lento será el movimiento ortodóntico; y cuanto mas intensa es la fuerza menor será la velocidad de migración dentaria.

- La presencia de vasodilatación y neutrófilos en áreas compresivas del ligamento periodontal, sugieren adecuada reacción inflamatoria con síntesis de prostaglandinas y cininas que propicia cambios en la actividad metabólica, importante en los procesos remodeladores

En el lado de fensión del ligamento periodontal, las células mesenquimales se transforman en osteoblastos y fibroblastos formadores respectivamente en tejido óseo y fibras de colágeno.

- El cemento y la dentina radicular también se remodelan durante la aplicación de fuerzas ortodónticas leves y continuas.

\section{Referencias}

1.Vellini Ferreira, Flavio. Ortodoncia Diagnóstico y Planificación Clinica. editorial Artes Medicas latinoamericanas. $1^{\circ}$ edición. Sao Patulo - Brasil. 2002: 361 - 374 .

2.R. J. Waddington and G. Embery. Proteoglycans and Or-thoderntic Tooth Muvement fournul of Orthodontics 2001; 28 (4):281-290.

3.Martina Von Böhl, DDS; Jaap Maltha, PhD; Hans Von den Hoff, PhD; Anne Marie KuijpersJagtman, DDS, PhD, FDSRCSEng.
Changes in the Periodontal Ligamext After Experi-mental Tooth Movement Using High and Low Continuous Forces in Beagle Dogs. The Angle Orthodontist.2004; 74 (1): 16-25.

4.Ten Cate. Histologia Oral $2^{\circ}$ ed Madrid Ed Panamericana. 1986;345-250.

5.Pilon JIGM, Kuijpers-Jagtman AM, Maltha IC. Magnitude of orthodontic forces and rate of bodily footh movement: an experimental study in beagle dogs. Am I Orthod Dentofrcial Orthop 1996;110:16-23.

6.Quinn R, Yoshikawa D. A reassessment of force magnitude in orthodontics. Am I Orthod. $1985 ; 88 ; 252-260$

7.Lee BW. Relationship between toothmovement rate and estimated pressure applied. I Dent Res. 1964. 44:1053

8.Hixon EH, Atiksan H, Callow GE, McDonald HW. Optimal force, differential force, and anchorage. Am I Orthod. 1969; 55:437-57.

9.Katona TR, Paydar NH, Akay HU, Roberts WE. Stress analysis of bone modeling response to rat molat orthodontics. I Bromech. 1995; 28:2738.

10.Row KL, Johnson RB. Distribution of $3 \mathrm{H}$-proline within transseptal fibers of the rat following release of orthodontic forces. Am I Anat. 1990;189:179-188.

11.Ren $Y$, Maltha $\mathrm{JC}_{5}$ KuijpersJagtman AM. Optimum force magnitude for orthodontic tooth movement: a systematic literature review. Angle Orthod. 2003; 73:86-92. 